\title{
DIMENSIONAL CHARACTERISTICS OF INVARIANT MEASURES FOR CIRCLE DIFFEOMORPHISMS
}

\author{
VICTORIA SADOVSKAYA*
}

\begin{abstract}
We consider pointwise, box, and Hausdorff dimensions of invariant measures for circle diffeomorphisms. We discuss the cases of rational, Diophantine, and Liouville rotation numbers. Our main result is that for any Liouville number $\tau$ there exists a $C^{\infty}$ circle diffeomorphism with rotation number $\tau$ such that the pointwise and box dimensions of its unique invariant measure do not exist. Moreover, the lower pointwise and lower box dimensions can equal any value $0 \leq \beta \leq 1$.
\end{abstract}

\section{INTRODUCTION}

The study of dimensional characteristics of invariant sets and measures was originated by physicists and applied mathematicians in the context of strange attractors. Beginning with the work of Eckmann and Ruelle 3 it developed into a rigorous mathematical theory. Dimension theory now plays an important role in dynamics [10]. Dimensional properties of invariant sets and measures are often related to other characteristics of the dynamical system, such as Lyapunov exponents and entropy.

In this paper we study pointwise, box, and Hausdorff dimensions of invariant measures for circle diffeomorphisms. The notion of pointwise (or local) dimension was introduced by Young in [11]. It plays an important role in dimension theory of dynamical systems. For a Borel measure $\mu$ on a metric space $X$, its lower and upper pointwise dimensions at a point $x$ are defined as

$$
\underline{d}_{\mu}(x)=\liminf _{r \rightarrow 0} \frac{\log \mu(B(x, r))}{\log r} \quad \text { and } \quad \bar{d}_{\mu}(x)=\limsup _{r \rightarrow 0} \frac{\log \mu(B(x, r))}{\log r},
$$

where $B(x, r)$ is a ball of radius $r$ centered at $x$. If the two limits coincide, then their common value $d_{\mu}(x)$ is called the pointwise dimension of $\mu$ at $x$. The pointwise dimension describes the local distribution of the measure and of a typical orbit. It serves as an important tool for estimating the Hausdorff and box dimensions of measures and sets, see Section 2.2. For example, if the pointwise dimension of an ergodic measure $\mu$ exists almost everywhere, then all dimensional characteristics of $\mu$ coincide and give a fundamental characteristic of $\mu$ called the fractal dimension.

Date: August 31, 2008.

* Supported in part by NSF grant DMS-0401014. 
In 22 Barreira, Pesin, and Schmeling showed that pointwise dimension exits for any hyperbolic invariant measure of a $C^{1+\alpha}$ diffeomorphism. Dimensional structures of non-hyperbolic measures can be more complicated. In [9] Ledrappier and Misiurewicz constructed an example of a $C^{r}$ map of an interval preserving an ergodic measure whose pointwise dimension does not exist almost everywhere. A natural class of non-hyperbolic measures is given by invariant measures for circle diffeomorphisms with irrational rotation numbers. In [8] we constructed examples of such diffeomorphisms for which pointwise dimension of the measures does not exist almost everywhere.

Dimensional properties of an invariant measure of a circle diffeomorphism $f$ depend significantly on the rotation number of $f$ (see Section 2.1). First we consider the simpler cases of rational and Diophantine numbers, and then describe our main result for Liouville numbers. The rotation number of $f$ is rational if and only if $f$ has periodic points. Such a diffeomorphism may preserve a variety of measures with different properties, however, any ergodic invariant measure for $f$ is a uniform $\delta$-measure on a periodic orbit. This immediately implies the following result.

Proposition 1.1. Let $f$ be a circle homeomorphism with a rational rotation number and let $\mu$ be an ergodic invariant measure for $f$. Then

(1) $d_{\mu}(x)=0$ for $\mu$-almost every $x$ in $S^{1}$,

(2) $\operatorname{dim}_{H} \mu=\underline{\operatorname{dim}}_{B} \mu=\overline{\operatorname{dim}}_{B} \mu=0$.

In contrast, diffeomorphisms with an irrational rotation number are uniquely ergodic. In this case, the properties of the invariant measure depend on how well the irrational rotation number can be approximated by rational numbers. The numbers that can not be rapidly approximated by rationals are called Diophantine.

Definition 1.2. A number $\tau$ is called Diophantine if there exist $\delta>0$ and $K>0$ such that

$$
|\tau-p / q|>K /|q|^{2+\delta} \text { for any integers } p \text { and } q \text {. }
$$

Circle diffeomorphisms with Diophantine rotation numbers are smoothly conjugate to rotations. Therefore the invariant measure for such a diffeomorphism is equivalent to the Lebesgue measure and hence has the same dimensional properties.

Proposition 1.3. Let $f$ be $a C^{\infty}$ circle homeomorphism with a Diophantine rotation number. Then for its unique invariant measure $\mu$,

(1) $d_{\mu}(x)=1$ for every $x$ in $S^{1}$,

(2) $\operatorname{dim}_{H} \mu=\underline{\operatorname{dim}}_{B} \mu=\overline{\operatorname{dim}}_{B} \mu=1$. 
The most interesting case is that of Liouville rotation numbers. These are irrational numbers that can be rapidly approximated by rationals, more precisely:

Definition 1.4. An irrational number $\tau$ is called a Liouville number if for any $n \geq 1$ there exist integers $p$ and $q, q>1$, such that

$$
|\tau-p / q|<1 / q^{n} \text {. }
$$

Clearly, an irrational number is Liouville if and only if it is not Diophantine. The set of all Liouville numbers is a dense $G_{\delta}$ set in $\mathbb{R}$, and it has zero Lebesgue measure. Our main result, Theorem 1.5, shows that in the case of a Liouville rotation number different types of dimensional properties of the invariant measure are realized. In particular, pointwise and box dimensions may not exist, which is in contrast to the Diophantine case as well as to the case of hyperbolic measures.

Theorem 1.5. Let $\tau$ be a Liouville number and let $0 \leq \beta \leq 1$. There exists a $C^{\infty}$ circle diffeomorphism $f$ with rotation number $\tau$ such that for its unique invariant measure $\mu$,

(1) $\underline{d}_{\mu}(x)=\beta$ and $\bar{d}_{\mu}(x)=1$ for $\mu$-almost every $x$ in $S^{1}$,

(2) $\operatorname{dim}_{H} \mu=\underline{\operatorname{dim}}_{B} \mu=\beta$ and $\overline{\operatorname{dim}}_{B} \mu=1$.

It is an interesting open question whether there exists a circle diffeomorphism with irrational rotation number whose invariant measure has upper pointwise dimension less than 1 on a set of positive measure.

Our constructions are based on a method developed by Anosov and Katok in [1] to produce examples of diffeomorphisms with specific ergodic properties. In [8] we used this method to construct examples of diffeomorphisms satisfying (1) and (2) of Theorem 1.5. However, the qualitative nature of the arguments did not allow us to construct examples for a given rotation number, or even describe explicitly the rotation numbers in our examples. In this paper we use some ideas developed in [4, 5] to make an explicit construction with specific quantitative estimates. This allows us to produce the examples for all Liouville rotation numbers.

We note that any $C^{2}$ circle diffeomorphism $f$ with irrational rotation number is topologically conjugate to the corresponding rotation. The conjugacy gives the distribution function of the invariant measure $\mu$. In the theorem above, $\mu$ is singular for $\beta<1$, and so is the conjugacy. Thus the theorem implies that for any Liouville rotation number there exists a diffeomorphisms with singular conjugacy. Similar methods may be used to construct diffeomorphisms with specific degree of regularity of the conjugacy for any Liouville rotation number. 


\section{PRELIMINARIES}

2.1. Rotation number of a circle homeomorphism. (See [7] for more details.) Let $f$ be an orientation-preserving homeomorphism of $S^{1}$, let $\pi: \mathbb{R} \rightarrow S^{1}=\mathbb{R} / \mathbb{Z}$ be the natural projection, and let $F$ be a homeomorphism of $\mathbb{R}$ such that $f \circ \pi=\pi \circ F$. Then the following limit exists and has the same value for all $x$ :

$$
\tau(F)=\lim _{|n| \rightarrow \infty} \frac{1}{n}\left(F^{n}(x)-x\right) .
$$

The number $\tau(f)=\pi(\tau(F))$ is called the rotation number of $f$. If $h: S^{1} \rightarrow S^{1}$ is a homeomorphism, then $\tau\left(h^{-1} \circ f \circ h\right)=\tau(f)$. In particular, if $f$ is topologically conjugate to a rotation by $\tau$ then $f$ has rotation number $\tau$.

2.2. Hausdorff and box dimensions of sets and measures. (See [10] for more details.) The upper and lower box dimensions of a set $Z \subset \mathbb{R}^{k}$ are defined as

$$
\overline{\operatorname{dim}}_{B} Z=\limsup _{\varepsilon \rightarrow 0} \frac{\log N(Z, \varepsilon)}{\log (1 / \varepsilon)} \quad \text { and } \quad \underline{\operatorname{dim}}_{B} Z=\liminf _{\varepsilon \rightarrow 0} \frac{\log N(Z, \varepsilon)}{\log (1 / \varepsilon)},
$$

where $N(Z, \varepsilon)$ is the least number of balls of diameter $\varepsilon$ needed to cover $Z$.

For a number $\alpha \geq 0$, the $\alpha$-Hausdorff measure of $Z$ is

$$
m_{H}(Z, \alpha)=\lim _{\varepsilon \rightarrow 0} \inf _{\mathcal{G}} \sum_{U \in \mathcal{G}}(\operatorname{diam} U)^{\alpha},
$$

where the infimum is taken over all finite or countable coverings $\mathcal{G}$ of $Z$ by open sets with diameter at most $\varepsilon$. The Hausdorff dimension of $Z$ is

$$
\operatorname{dim}_{H} Z=\inf \left\{\alpha: m_{H}(Z, \alpha)=0\right\}=\sup \left\{\alpha: m_{H}(Z, \alpha)=\infty\right\} .
$$

The Hausdorff and upper and lower box dimensions of a Borel probability measure $\mu$ are defined as follows:

$$
\begin{aligned}
& \operatorname{dim}_{H} \mu=\inf \left\{\operatorname{dim}_{H} Z: \mu(Z)=1\right\}, \\
& \underline{\operatorname{dim}}_{B} \mu=\lim _{\varepsilon \rightarrow 0} \inf \left\{\underline{\operatorname{dim}}_{B} Z: \mu(Z)>1-\varepsilon\right\}, \\
& \overline{\operatorname{dim}}_{B} \mu=\lim _{\varepsilon \rightarrow 0} \inf \left\{\overline{\operatorname{dim}}_{B} Z: \mu(Z)>1-\varepsilon\right\} .
\end{aligned}
$$

It is known that $\operatorname{dim}_{H} \mu \leq \underline{\operatorname{dim}}_{B} \mu \leq \overline{\operatorname{dim}}_{B} \mu$.

The following result by L.-S. Young [11] shows how the pointwise dimension of a measure can be used to estimate its box and Hausdorff dimensions.

Theorem 2.1. Let $\mu$ be a Borel finite measure on $\mathbb{R}^{m}$. Then

(1) If $\underline{d}_{\mu}(x) \geq d$ for $\mu$-almost every $x$ then $\operatorname{dim}_{H} \mu \geq d$;

(2) If $\bar{d}_{\mu}(x) \leq d$ for $\mu$-almost every $x$ then $\overline{\operatorname{dim}}_{B} \mu \leq d$;

(3) If $\underline{d}_{\mu}(x)=\bar{d}_{\mu}(x)=d$ for $\mu$-almost every $x$, then

$\operatorname{dim}_{H} \mu=\underline{\operatorname{dim}}_{B} \mu=\overline{\operatorname{dim}}_{B} \mu=d$. 


\section{Proofs}

Throughout this paper we will identify the unit circle $S^{1}=\mathbb{R} / \mathbb{Z}$ with the interval $[0,1]$. Let $\tau$ be an irrational number and let $R_{\tau}$ be the rotation by $\tau$. The Lebesgue measure is the only measure preserved by $R_{\tau}$. Suppose that

$$
f=h^{-1} \circ R_{\tau} \circ h,
$$

where $h$ is a homeomorphism. Then the unique invariant measure $\mu$ for $f$ is the push-forward of the Lebesgue measure $\lambda$ by $h^{-1}$, i.e. $\mu(A)=\lambda(h A)$. This means that $h$ is the distribution function for $\mu$, i.e.

$$
\mu\left(\left[x_{1}, x_{2}\right)\right)=h\left(x_{2}\right)-h\left(x_{1}\right)
$$

for any interval $\left[x_{1}, x_{2}\right) \subset S^{1}$, and hence

$$
\mu(B(x, r))=\Delta h(x, r) \stackrel{\text { def }}{=} h(x+r)-h(x-r) .
$$

In particular, if $h$ is continuously differentiable, then for any $x$ and $r<1 / 2$,

$$
2 r \cdot \min _{[x-r, x+r]}\left|h^{\prime}\right| \leq \mu(B(x, r)) \leq 2 r \cdot \max _{[x-r, x+r]}\left|h^{\prime}\right| .
$$

3.1. Proof of Proposition 1.3. The following result was established by M.-R. Herman in [6]: any $C^{2+\varepsilon}$ circle diffeomorphism whose rotation number $\tau$ satisfies the Diophantine condition (1.1) with some $K>0$ and $0<\delta<\varepsilon$ is conjugate to the rotation $R_{\tau}$ via a $C^{1}$ diffeomorphism. It follows that any $C^{\infty}$ diffeomorphism with a Diophantine rotation number is smoothly conjugate to the corresponding rotation. This implies that there exist constants $m$ and $M$ such that $2 m r \leq \mu(B(x, r)) \leq 2 M r$ for all $x$ and all $r<1 / 2$. Therefore, $d_{\mu}(x)=1$ for every $x \in S^{1}$, and hence $\operatorname{dim}_{H} \mu=\underline{\operatorname{dim}}_{B} \mu=\overline{\operatorname{dim}}_{B} \mu=1$ by Theorem 2.1.

3.2. Proof of Theorem 1.5. Let $\tau$ be a Liouville number. First we note that to obtain the result for the case of $\beta=1$ it suffices to take $f=R_{\tau}$. The rotation $R_{\tau}$ preserves the Lebesgue measure, which satisfies (1) and (2). From now on we will assume that $0 \leq \beta<1$.

We will obtain the diffeomorphism $f$ as a limit of a sequence of diffeomorphisms

$$
f_{n}=h_{n}^{-1} \circ R_{\tau_{n}} \circ h_{n}
$$

where $h_{n}$ are $C^{\infty}$ diffeomorphisms of $S^{1}$ and $\tau_{n}$ are rational numbers that converges to $\tau$. The sequences $\left\{h_{n}\right\}$ and $\left\{\tau_{n}\right\}$ will be defined inductively. Once $h_{n}$ is selected, we will construct $h_{n+1}$ in the form

$$
h_{n+1}=A_{n} \circ h_{n}, \quad \text { where } A_{n}=\mathrm{Id}+a_{n}
$$

is a diffeomorphism and $a_{n}$ is a $C^{\infty}$ periodic function.

The diffeomorphisms $f_{n}$ will converge in the $C^{\infty}$ topology, and $h_{n}$ as well as $h_{n}^{-1}$ will converge in $C^{0}$. The homeomorphism $h=\lim _{n \rightarrow \infty} h_{n}$ will give the distribution function of the invariant measure $\mu$ for $f$. 
We will use the following norm of diffeomorphisms and the corresponding distance.

Definition 3.1. Let $g$ be a $C^{n}$ diffeomorphism of $[0,1]$. We define a norm of $g$

$$
\|g\|_{n}^{*}=\max \left|g^{(i)}(x)\right| \text {, }
$$

where the maximum is taken over all $x$ and $0 \leq i \leq n$, and we denote

$$
\|g\|_{n}=\max \left\{\|g\|_{n}^{*},\left\|g^{-1}\right\|_{n}^{*}\right\} .
$$

For two $C^{n}$ diffeomorphisms $g_{1}$ and $g_{2}$, we set

$$
d_{n}\left(g_{1}, g_{2}\right)=\max \left\{\left\|g_{1}-g_{2}\right\|_{n}^{*},\left\|g_{1}^{-1}-g_{2}^{-1}\right\|_{n}^{*}\right\} .
$$

In the three lemmas below we will use Faà di Bruno's formula, which generalizes the chain rule to higher derivatives:

$$
\frac{d^{n}}{d x^{n}} f(g(x))=\sum c_{m_{1}, \ldots, m_{n}} f^{\left(m_{1}+\cdots+m_{n}\right)}(g(x)) \prod_{j=1}^{n}\left(g^{(j)}(x)\right)^{m_{j}},
$$

where the constants $c_{m_{1}, \ldots, m_{n}}$ depend only on $m_{1}, \ldots, m_{n}$, and the sum is taken over all $n$-tuples $\left(m_{1}, \ldots, m_{n}\right)$ of integers satisfying

$$
1 m_{1}+2 m_{2}+\cdots+n m_{n}=n \quad \text { and } \quad m_{i} \geq 0, \quad i=1, \ldots, n .
$$

The following lemma gives an estimate for the distance between two maps conjugate to two rotations via the same diffeomorphism.

Lemma 3.2. Let $R_{\tau_{1}}$ and $R_{\tau_{2}}$ be two circle rotations, and let $h$ be a $C^{n+1}$ circle diffeomorphism. Then

$$
d_{n}\left(h^{-1} \circ R_{\tau_{1}} \circ h, h^{-1} \circ R_{\tau_{2}} \circ h\right) \leq c_{n}\left|\tau_{1}-\tau_{2}\right| \cdot\|h\|_{n+1}^{n+1},
$$

where the constant $c_{n}$ depends only on $n$.

Proof. We will estimate $\max \left|\frac{d^{k}}{d x^{k}}\left(h^{-1} \circ R_{\tau_{1}} \circ h\right)-\frac{d^{k}}{d x^{k}}\left(h^{-1} \circ R_{\tau_{2}} \circ h\right)\right|$ for $0 \leq k \leq n$. For $k=0$ we have

$$
\max \left|h^{-1}\left(h(x)+\tau_{1}\right)-h^{-1}\left(h(x)+\tau_{2}\right)\right| \leq \max \left|\left(h^{-1}\right)^{\prime}\right| \cdot\left|\tau_{1}-\tau_{2}\right| \leq\|h\|_{1} \cdot\left|\tau_{1}-\tau_{2}\right| .
$$

For $1 \leq k \leq n$ formula (3.2) yields

$$
\begin{aligned}
& \frac{d^{k}}{d x^{k}}\left(h^{-1} \circ R_{\tau} \circ h\right)(x)=\frac{d^{k}}{d x^{k}}\left(h^{-1}(h(x)+\tau)\right) \\
& =\sum c_{m_{1}, \ldots, m_{k}}\left(h^{-1}\right)^{\left(m_{1}+\cdots+m_{k}\right)}(h(x)+\tau) \prod_{j=1}^{k}\left(h^{(j)}(x)\right)^{m_{j}} .
\end{aligned}
$$


We estimate the difference between the corresponding terms in $\frac{d^{k}}{d x^{k}}\left(h^{-1} \circ R_{\tau_{1}} \circ h\right)(x)$ and $\frac{d^{k}}{d x^{k}}\left(h^{-1} \circ R_{\tau_{2}} \circ h\right)(x)$. By (3.3) $), m_{1}+\cdots+m_{k} \leq k$, and we have

$$
\begin{aligned}
& \left|\prod_{j=1}^{k}\left(h^{(j)}(x)\right)^{m_{j}}\left(\left(h^{-1}\right)^{\left(m_{1}+\cdots+m_{k}\right)}\left(h(x)+\tau_{1}\right)-\left(h^{-1}\right)^{\left(m_{1}+\cdots+m_{k}\right)}\left(h(x)+\tau_{2}\right)\right)\right| \\
& \leq \max \left|\prod_{j=1}^{k}\left(h^{(j)}(x)\right)^{m_{j}}\right| \cdot \max \left|\left(h^{-1}\right)^{\left(m_{1}+\cdots+m_{k}+1\right)}\right| \cdot\left|\tau_{1}-\tau_{2}\right| \\
& \leq\|h\|_{k}^{k} \cdot\|h\|_{k+1} \cdot\left|\tau_{1}-\tau_{2}\right| \leq\|h\|_{k+1}^{k+1} \cdot\left|\tau_{1}-\tau_{2}\right| .
\end{aligned}
$$

It follows that for any $0 \leq k \leq n$,

$$
\max \left|\frac{d^{k}}{d x^{k}}\left(h^{-1} \circ R_{\tau_{1}} \circ h\right)(x)-\frac{d^{k}}{d x^{k}}\left(h^{-1} \circ R_{\tau_{2}} \circ h\right)(x)\right| \leq c_{n}\left|\tau_{1}-\tau_{2}\right| \cdot\|h\|_{n+1}^{n+1},
$$

where $c_{n}$ is the sum of the coefficients $c_{m_{1} \ldots m_{n}}$ in $(3.2)$. Since $\left(h^{-1} \circ R_{\tau} \circ h\right)^{-1}=$ $h^{-1} \circ R_{-\tau} \circ h$, we have the same estimate for the inverses of the functions, and (3.4) follows.

When constructing the diffeomorphism $h_{n+1}=A_{n} \circ h_{n}$, we will choose the function $A_{n}$ in a specific form, as in the following lemma.

Lemma 3.3. Let $s$ and $\delta$ be positive numbers such that $1 / s$ is an integer and $\delta<s / 2$. Then there exists a $C^{\infty}$ diffeomorphism $A=A_{s, \delta}$ of $[0,1]$ such that

(1) $A=I d+a$, where $a=a_{s, \delta}$ is a non-negative $C^{\infty}$ function of period $s$,

(2) $A(0)=0, A(\delta)=s-\delta$, and $A(s)=s$,

(3) $\delta /(2 s) \leq A^{\prime}(x) \leq 2 s / \delta$ for all $x$,

(4) for each $n \geq 0$ there exists a constant $\rho_{n}$ that does not depend on $\delta$ and $s$ such that $\|A\|_{n} \leq \rho_{n} / \delta^{n^{2}}$.

Conditions (1), (2), and (3) guarantee that $A$ is a diffeomorphism.

Proof. First we construct the function $a$ on the intervals $[0, \delta]$. Let $g$ be a $C^{\infty}$ function on $[0,1]$ such that $g(x)=0$ in a neighborhood $[0, \varepsilon)$ of $0, g(x)=1$ in a neighborhood $(1-\varepsilon, 1]$ of 1 , and $0 \leq g^{\prime}(x) \leq 2$ for all $x$. We obtain the function $a$ on $[0, \delta]$ by rescaling $g$ :

$$
a(x)=(s-2 \delta) g(x / \delta) \text { for } x \in[0, \delta] .
$$

Then $a(x)=0$ in a neighborhood of $0, a(x)=s-2 \delta$ in a neighborhood of $\delta$, and $0 \leq a^{\prime}(x) \leq 2(s-2 \delta) / \delta=2 s / \delta-4$. This implies that $A(x)=x$ in a neighborhood of $0, A(\delta)=s-\delta, A^{\prime}(x)=1$ in a neighborhood of $\delta$, and $1 \leq A^{\prime}(x) \leq 2 s / \delta-3 \leq 2 s / \delta$ for all $x$ in $[0, \delta]$. 
Now we obtain the graph of the function $A$ on $[\delta, s]$ by reflecting its graph on $[0, \delta]$ with respect to the line $y=s-x$, i.e.

$$
A(x)=s-A^{-1}(s-x) \text { for } x \text { in }[s-\delta, s] .
$$

It follows from the symmetry of the graph that $A(x)=x$ in a neighborhood of $s$ and $1 \geq A^{\prime}(x) \geq \delta / 2 s$ for all $x$ in $[s-\delta, s]$. On this interval, we set $a(x)=A(x)-x$. Clearly, $a(x) \geq 0$ for all $x$ and $a(x)=0$ in a neighborhood of $s$. Then we extend $a$ to $[0,1]$ by periodicity, and thus obtain $A=\mathrm{Id}+a$ on $[0,1]$. Thus we have constructed a function $A$ satisfying (1), (2), and (3).

Now we will verify (4) for $A$ on $[0, \delta]$. Then (3.5) will imply that (4) is also satisfied for $A$ on $[\delta, s]$. Since $\max _{[0, \delta]}\left|a^{(n)}\right| \leq \max _{[0,1]}\left|g^{(n)}\right| / \delta^{n}$, we have

$$
\max _{[0, \delta]}\left|A^{(n)}\right| \leq\left(\max _{[0,1]}\left|g^{(n)}\right|+1\right) / \delta^{n} \stackrel{\text { def }}{=} \kappa_{n} / \delta^{n} \quad \text { for any } n \geq 0 .
$$

Let $G$ be the inverse function for $\left.A\right|_{[0, \delta]}$. We will show using induction that for any $n \geq 0$ there exists a constant $\xi_{n}$ independent of $\delta$ and $s$ such that

$$
\max _{[0,1]}\left|G^{(n)}\right| \leq \xi_{n} / \delta^{n^{2}} \quad \text { and } \quad \xi_{n} \geq \xi_{n-1} \geq \cdots \geq \xi_{0} .
$$

Clearly, $G(x) \leq 1$ and $G^{\prime}(x) \leq 1 \leq 1 / \delta$ for all $x$. Thus the statement holds for $k=0$ and $k=1$. Suppose that it holds for all $0 \leq k \leq n-1$. For $n \geq 2$, $\frac{d^{n}}{d x^{n}} G(A(x))=\frac{d^{n}}{d x^{n}} x=0$, and it follows from (3.2) that

$$
G^{(n)}(A(x)) \cdot\left(A^{\prime}(x)\right)^{n}=-\sum c_{m_{1}, \ldots, m_{n}} G^{\left(m_{1}+\cdots+m_{n}\right)}(A(x)) \prod_{j=1}^{n}\left(A^{(j)}(x)\right)^{m_{j}},
$$

where the sum is taken over all $n$-tuples $\left(m_{1}, \ldots m_{n}\right)$ such that $1 m_{1}+\cdots+n m_{n}=n$ and $m_{1} \neq n$. This implies that $m_{1}+\cdots+m_{n} \leq n-1$, and hence

$$
\left|G^{\left(m_{1}+\cdots+m_{n}\right)}(A(x))\right| \leq \xi_{n-1} / \delta^{(n-1)^{2}}
$$

by the induction assumption. Also,

$$
\left|\prod_{j=1}^{n}\left(A^{(j)}(x)\right)^{m_{j}}\right| \leq \prod_{j=1}^{n}\left(\kappa_{j} / \delta^{j}\right)^{m_{j}}=\left(\prod_{j=1}^{n} \kappa_{j}^{m_{j}}\right) / \delta^{1 m_{1}+2 m_{2}+\cdots+n m_{n}} \leq\left(\prod_{j=1}^{n} \kappa_{j}^{n}\right) / \delta^{n} .
$$

Using the estimates above, we obtain

$$
\left|G^{(n)}(A(x)) \cdot\left(A^{\prime}(x)\right)^{n}\right| \leq \xi_{n} / \delta^{(n-1)^{2}+n} \leq \xi_{n} / \delta^{n^{2}},
$$

where $\xi_{n} \geq \xi_{n-1}$ is a constant independent of $s$ and $\delta$. Since $A^{\prime}(x) \geq 1$ on $[0, \delta]$, it follows that

$$
\left|G^{(n)}(A(x))\right| \leq \xi_{n} / \delta^{n^{2}} \quad \text { for all } x \in[0, \delta] .
$$

Let $\rho_{n}=\max \left\{\kappa_{0}, \ldots, \kappa_{n}, \xi_{n}\right\}$, where $\kappa_{0}, \ldots \kappa_{n}$ are as in (3.6). Then

$$
\|A\|_{n}^{*} \leq \rho_{n} / \delta^{n} \leq \rho_{n} / \delta^{n^{2}}, \quad\left\|A^{-1}\right\|_{n}^{*}=\|G\|_{n}^{*} \leq \rho_{n} / \delta^{n^{2}}, \text { and thus }\|A\|_{n} \leq \rho_{n} / \delta^{n^{2}}
$$


Lemma 3.4. Let $A=A_{s, \delta}$ be a function as in Lemma 3.3. Then for any $n \geq 0$ and any $C^{n}$ diffeomorphism $h$

$$
\|A \circ h\|_{n} \leq \tilde{c}(h, n) / \delta^{n^{2}}
$$

where the constant $\tilde{c}(h, n)$ depends on $h$ and $n$, but not on $\delta$ and $s$.

Proof. Let $0 \leq k \leq n$. Each term of the sum representing $(A \circ h)^{(k)}$ is a product of a derivative of $A$ of order at most $k$ and at most $k$ derivatives of $h$, see (3.2). Therefore, each term can be estimated by $\|A\|_{k} \cdot\|h\|_{k}^{k} \leq\|A\|_{n} \cdot\|h\|_{n}^{n}$, and hence

$$
\|A \circ h\|_{n}^{*} \leq c_{n}\|A\|_{n} \cdot\|h\|_{n}^{n} \leq c_{n}\|h\|_{n}^{n} \cdot \rho_{n} / \delta^{n^{2}}
$$

where $c_{n}$ is the sum of the constants $c_{m_{1}, \ldots, m_{n}}$ in (3.2) and $\rho_{n}$ is as in Lemma $3.3(4)$. Each term of $\left(h^{-1} \circ A^{-1}\right)^{(k)}$ can be estimated as follows:

$$
\begin{aligned}
& \left|\left(h^{-1}\right)^{\left(m_{1}+\cdots+m_{k}\right)}\left(A^{-1}(x)\right) \prod_{j=1}^{k}\left(\left(A^{-1}\right)^{(j)}(x)\right)^{m_{j}}\right| \leq\|h\|_{k} \cdot\left(\rho_{1} / \delta^{1^{2}}\right)^{m_{1}} \ldots\left(\rho_{k} / \delta^{k^{2}}\right)^{m_{k}} \\
& \leq\|h\|_{k} \cdot \rho_{1} \ldots \rho_{k} / \delta^{1^{2} m_{1}+2^{2} m_{2}+\cdots+k^{2} m_{k}} \leq\|h\|_{k} \cdot \rho_{1} \ldots \rho_{k} / \delta^{k^{2}} \leq\|h\|_{n} \cdot \rho_{1} \ldots \rho_{n} / \delta^{n^{2}}
\end{aligned}
$$

since $1^{1} m_{1}+2^{2} m_{2}+\cdots+k^{2} m_{k} \leq k\left(1 m_{1}+2 m_{2}+\cdots+k m_{k}\right)=k^{2}$. It follows that

$$
\left\|h^{-1} \circ A^{-1}\right\|_{n}^{*} \leq c_{n}\|h\|_{n} \cdot \rho_{1} \ldots \rho_{n} / \delta^{n^{2}} .
$$

Thus $\|A \circ h\|_{n} \leq \tilde{c}(h, n) / \delta^{n^{2}}$.

For the rest of the proof we will consider the cases of $\beta=0$ and of $0<\beta<1$ separately.

The case of $\beta=0$.

We will construct the sequences $\left\{\tau_{n}\right\}_{n=1}^{\infty}$ and $\left\{h_{n}\right\}_{n=1}^{\infty}$ inductively. Let $h_{1}$ be the identity map, $\tau_{1}$ be a rational number close to $\tau$, and $s_{1}=1 / 2$. Suppose that a number $\tau_{n-1}=p_{n-1} / q_{n-1}$, a function $a_{n-1}$ of period $s_{n-1}$, and hence diffeomorphisms $A_{n-1}=\mathrm{Id}+a_{n-1}$ and $h_{n}=A_{n-1} \circ h_{n-1}$ are selected. We denote

$$
M_{n}=\max _{[0,1]} h_{n}^{\prime} \quad \text { and } \quad m_{n}=\min _{[0,1]} h_{n}^{\prime}=1 / \max _{[0,1]}\left(h_{n}^{-1}\right)^{\prime}
$$

Clearly, $M_{n} \geq 1$ and $0<m_{n} \leq 1$ for all $n \geq 1$, and $M_{n} \rightarrow \infty, m_{n} \rightarrow 0$ as $n \rightarrow \infty$.

We choose a rational number $\tau_{n}=p_{n} / q_{n}$, numbers $s_{n}$ and $\delta_{n}$, a function $a_{n}$ of a period $s_{n}$, and a function $A_{n}$ such that 
(i) $\left|\tau-\tau_{n}\right| \leq\left|\tau-\tau_{n-1}\right|$,

(ii) $\left|\tau-\tau_{n}\right|=\left|\tau-p_{n} / q_{n}\right| \leq 1 / q_{n}^{3 n^{4}}$,

(iii) $q_{n} \geq \max \left\{1 / s_{n-1}, 1 / m_{n},\left(3 M_{n}\right)^{n}, c_{n}, \tilde{c}\left(h_{n}, n+1\right)\right\}$,

where $c_{n}$ is as in (3.4) and $\tilde{c}\left(h_{n}, n+1\right)$ is as in (3.7),

(iv) $s_{n}=s_{n-1} / q_{n}$, hence $s_{n} \leq s_{n-1}^{2}$ and $s_{n} \leq 1 / 2^{n}$,

(v) $\delta_{n}=s_{n}^{n}$, in particular, $\delta_{n}<s_{n} / 2$,

(vi) $a_{n}$ and $A_{n}$ are as in Lemma 3.3 with $\delta=\delta_{n}$ and $s=s_{n}$.

Conditions (i), (ii), and (iii) can be satisfied since $\tau$ is a Liouville number. Condition (iv) ensures that the maps $A_{n}$ and $R_{\tau_{n}}$ commute, and hence

$$
\begin{aligned}
& h_{n}^{-1} \circ R_{\tau_{n}} \circ h_{n}=h_{n}^{-1} \circ A_{n}^{-1} \circ A_{n} \circ R_{\tau_{n}} \circ h_{n} \\
& =h_{n}^{-1} \circ A_{n}^{-1} \circ R_{\tau_{n}} \circ A_{n} \circ h_{n}=\left(A_{n} \circ h_{n}\right)^{-1} \circ R_{\tau_{n}} \circ\left(A_{n} \circ h_{n}\right)
\end{aligned}
$$

Conditions (iii), (iv), and (v) imply that $1 / \delta_{n}=1 / s_{n}^{n}=q_{n}^{n} / s_{n-1}^{n} \leq q_{n}^{2 n}$. Using this as well as (3.4) and (3.7) we obtain

$$
\begin{aligned}
& d_{n}\left(f_{n+1}, f_{n}\right)=d_{n}\left(h_{n+1}^{-1} \circ R_{\tau_{n+1}} \circ h_{n+1}, h_{n}^{-1} \circ R_{\tau_{n}} \circ h_{n}\right) \\
& =d_{n}\left(\left(A_{n} \circ h_{n}\right)^{-1} \circ R_{\tau_{n+1}} \circ\left(A_{n} \circ h_{n}\right), \quad\left(A_{n} \circ h_{n}\right)^{-1} \circ R_{\tau_{n}} \circ\left(A_{n} \circ h_{n}\right)\right) \\
& \leq c_{n}\left|\tau_{n+1}-\tau_{n}\right| \cdot\left\|A_{n} \circ h_{n}\right\|_{n+1}^{n+1} \leq 2 c_{n}\left|\tau-\tau_{n}\right| \cdot\left(\tilde{c}\left(h_{n}, n+1\right) / \delta_{n}^{(n+1)^{2}}\right)^{n+1} \\
& \leq 2 q_{n}\left(1 / q_{n}^{3 n^{4}}\right)\left(q_{n} \cdot q_{n}^{2 n(n+1)^{2}}\right)^{n+1} \leq 2\left(1 / q_{n}^{3 n^{4}}\right) q_{n}^{2 n(n+1)^{3}+n+2} \leq 1 / 2^{n}
\end{aligned}
$$

for all sufficiently large $n$. Since $d_{n}\left(f_{m+1}, f_{m}\right) \leq d_{m}\left(f_{m+1}, f_{m}\right) \leq 1 / 2^{m}$ for $m \geq n$, it follows that the sequence $\left\{f_{n}\right\}$ converges in the $C^{n}$-topology for any $n$, i.e. it converges in the $C^{\infty}$ topology.

Now we will establish the convergence of the diffeomorphisms $h_{n}$. We recall that $A_{n}=\mathrm{Id}+a_{n}$ is a diffeomorphism, where $a_{n}$ is a $C^{\infty}$ function of period $s_{n}$, and $1 / s_{n}$ is an integer. It follows that $\max _{[0,1]}\left|A_{n}-\mathrm{Id}\right| \leq s_{n}$ and $\max _{[0,1]}\left|A_{n}^{-1}-\mathrm{Id}\right| \leq s_{n}$. Since $h_{n+1}=A_{n} \circ h_{n}$, we estimate

$$
\begin{gathered}
\max _{[0,1]}\left|h_{n+1}-h_{n}\right|=\max _{[0,1]}\left|\left(A_{n}-\mathrm{Id}\right) \circ h_{n}\right| \leq s_{n}, \quad \text { and } \\
\max _{[0,1]}\left|h_{n+1}^{-1}-h_{n}^{-1}\right|=\max _{[0,1]}\left|h_{n}^{-1} \circ\left(A_{n}^{-1}-\mathrm{Id}\right)\right| \leq \max _{[0,1]}\left(h_{n}^{-1}\right)^{\prime} \cdot s_{n}=\left(1 / m_{n}\right) s_{n} \leq s_{n-1}
\end{gathered}
$$

by (3.8) (iii) and (iv). This implies that $d_{0}\left(h_{n+1}, h_{n}\right) \leq s_{n-1}$, and since $s_{n} \leq 1 / 2^{n}$, it follows that the sequence of diffeomorphisms $\left\{h_{n}\right\}$ converges to a homeomorphism $h$ with respect to the distance $d_{0}$. Moreover, since $s_{n} \leq s_{n-1}^{2}$ we have

$$
\max _{[0,1]}\left|h-h_{n}\right| \leq \sum_{k=n}^{\infty} s_{k} \leq 2 s_{n}
$$


Now we will prove the dimensional properties of the invariant measure $\mu$ with distribution function $h$. By the construction, most of the growth of $A_{n}$ on the interval $[0,1]$ is concentrated on the union of intervals $\left[i s_{n}, i s_{n}+\delta_{n}\right]$. Let

$$
\tilde{E}_{n}=\bigcup_{i=0}^{\left(1 / s_{n}\right)-1}\left[i s_{n}, i s_{n}+\delta_{n}\right] \text { and } E_{n}=h_{n}^{-1}\left(\tilde{E}_{n}\right) .
$$

Then $h_{n+1}\left(E_{n}\right)=\left(A_{n} \circ h_{n}\right)\left(h_{n}^{-1}\left(\tilde{E}_{n}\right)\right)=A_{n}\left(\tilde{E}_{n}\right)$.

Since $\tilde{E}_{n}$ consists of $1 / s_{n}$ intervals $\left[i s_{n}, i s_{n}+\delta_{n}\right]$ and $A\left(i s_{n}+\delta_{n}\right)-A\left(i s_{n}\right)=s_{n}-\delta_{n}$, the total growth of $A_{n}$ on $\tilde{E}_{n}$ is

$$
\left(s_{n}-\delta_{n}\right)\left(1 / s_{n}\right)=1-\delta_{n} / s_{n}=1-s_{n}^{n} / s_{n} \geq 1-s_{n}^{n-1} \geq 1-s_{n},
$$

and the total growth of $h_{n+1}$ on $E_{n}$ is the same. By (3.10), $\Delta h \geq \Delta h_{n}-4 s_{n+1}$ on each of the $1 / s_{n}$ intervals in $E_{n}$. Since $s_{n+1} \leq s_{n}^{2}$ and $s_{n} \leq 1 / 2^{n}$ by (3.8) (iv), we estimate that the total growth of $h$ on the set $E_{n}$ is at least

$$
1-s_{n}-4 s_{n+1}\left(1 / s_{n}\right) \geq 1-s_{n}-4 s_{n}^{2} / s_{n}=1-5 s_{n} \geq 1-5 / 2^{n} \text {. }
$$

Thus, for the measure $\mu$ with distribution function $h$,

$$
\mu\left(E_{n}\right) \geq 1-5 / 2^{n} \text {. }
$$

Now we will show that $\underline{d}_{\mu}(x)$, the lower pointwise dimension of $\mu$ at $x$, is 0 for $\mu$-almost every $x$. We recall that $m_{n}=\min _{[0,1]} h_{n}^{\prime}$. The length of each interval $I$ in the set $E_{n}$ is bounded above by $\delta_{n} / m_{n}$, since the length of $h_{n}(I)$ is $\delta_{n}$. Let

$$
r_{n}=\delta_{n} / m_{n}=s_{n}^{n} / m_{n} \text {. }
$$

Let $x$ be a point in $E_{n}$. Then the interval $\left[x-r_{n}, x+r_{n}\right]$ contains one of the intervals in $E$ and hence $\Delta h_{n+1}\left(x, r_{n}\right) \geq s_{n}-\delta_{n}$. It follows from (3.10) that

$$
\Delta h\left(x, r_{n}\right) \geq \Delta h_{n+1}\left(x, r_{n}\right)-4 s_{n+1} \geq s_{n}-\delta_{n}-4 s_{n+1} \geq s_{n} / 2
$$

for all sufficiently large $n$ since $\delta_{n}=s_{n}^{n}$ and $s_{n+1} \leq s_{n}^{2}$. Therefore

$$
\frac{\log \Delta h\left(x, r_{n}\right)}{\log r_{n}} \leq \frac{\log \left(s_{n} / 2\right)}{\log r_{n}}=\frac{\log \left(s_{n} / 2\right)}{\log \left(s_{n}^{n} / m_{n}\right)} \leq \frac{2}{n} .
$$

The last inequality is equivalent to $s_{n} \leq m_{n}^{2 / n} / 2$, and it follows from (3.8) that $s_{n}=s_{n-1} / q_{n} \leq s_{n-1} m_{n} \leq m_{n} / 2$.

Thus for any sufficiently large $n$ there exists $r_{n}>0$ such that

$$
\frac{\log \mu\left(B\left(x, r_{n}\right)\right)}{\log r_{n}}=\frac{\log \Delta h\left(x, r_{n}\right)}{\log r_{n}} \leq \frac{2}{n} \quad \text { for any } x \in E_{n}
$$

and $r_{n} \rightarrow 0$ as $n \rightarrow \infty$. Let $x$ be a point in $[0,1]$. If follows that $\underline{d}_{\mu}(x)=0$ provided that for any $m$ there exist $n \geq m$ such that $x \in E_{n}$. Otherwise, $x$ is in $J=\bigcup_{m=1}^{\infty} \bigcap_{n=m}^{\infty}\left([0,1]-E_{n}\right)$. It follows from (3.12) that $\mu\left(\bigcap_{n=m}^{\infty}\left([0,1]-E_{n}\right)\right)=0$ and hence $\mu(J)=0$. We conclude that

$$
\underline{d}_{\mu}(x)=0 \quad \text { for } \mu \text {-almost every } x \in S^{1} .
$$


Now we will show that $\bar{d}_{\mu}(x)$, the upper pointwise dimension of $\mu$ at $x$, equals 1 for $\mu$-almost all $x$. We recall that $M_{n}=\max _{[0,1]} h_{n}^{\prime}$, and hence $\Delta h_{n}(x, r) \leq 2 r M_{n}$. We take $\tilde{r}_{n}=\left(3 M_{n}\right)^{-n}$ and note that by (3.8) $s_{n}=s_{n-1} / q_{n}<1 / q_{n} \leq 1 /\left(3 M_{n}\right)^{n} \leq \tilde{r}_{n}$. It follows that

$$
\Delta h\left(x, \tilde{r}_{n}\right) \leq \Delta h_{n}\left(x, \tilde{r}_{n}\right)+4 s_{n} \leq 2 \tilde{r}_{n} M_{n}+4 \tilde{r}_{n} \leq 3 M_{n} \tilde{r}_{n}
$$

for all sufficiently large $n$. Hence for all $x$,

$$
\frac{\log \mu\left(B\left(x, \tilde{r}_{n}\right)\right)}{\log \tilde{r}_{n}}=\frac{\log \Delta h\left(x, \tilde{r}_{n}\right)}{\log \tilde{r}_{n}} \geq \frac{\log \left(3 M_{n} \tilde{r}_{n}\right)}{\log \tilde{r}_{n}}=1+\frac{\log \left(3 M_{n}\right)}{\log \left(\left(3 M_{n}\right)^{-n}\right)}=1-\frac{1}{n} .
$$

Clearly, $\tilde{r}_{n} \rightarrow 0$ as $n \rightarrow \infty$, and we conclude that $\bar{d}_{\mu}(x) \geq 1$ for all $x$. Since $\mu$ is a Borel probability measure on $S^{1}, \bar{d}_{\mu}(x) \leq 1$ for $\mu$-almost every $x$ ([8] Lemma 2.1). Thus $\bar{d}_{\mu}(x)=1$ for $\mu$-almost all $x$. Combining this with (3.13) we obtain

$$
\underline{d}_{\mu}(x)=0 \quad \text { and } \quad \bar{d}_{\mu}(x)=1 \quad \text { for } \mu \text {-almost every } x \text {. }
$$

This completes the proof of the first statement of Theorem 1.5 for the case of $\beta=0$.

Now we will prove the results for the box and Hausdorff dimensions of $\mu$ (see Section 2.2 for the definitions). First we show that $\overline{\operatorname{dim}}_{B} \mu=1$. For $\tilde{r}_{n}$ as above, we have

$$
\mu\left(B\left(x, \tilde{r}_{n}\right)\right) \leq \tilde{r}_{n}^{1-1 / n} \text { for any } x \in S^{1} .
$$

Let $Z$ be a set in $S^{1}$ with $\mu(Z)>0$. Then at least $\mu(Z) \cdot \tilde{r}_{n}^{-(1-1 / n)}$ balls of radius $\tilde{r}_{n}$ are needed to cover $Z$. Thus,

$$
\frac{\log N\left(Z, \tilde{r}_{n}\right)}{\log \left(1 / \tilde{r}_{n}\right)} \geq \frac{\log \left(\mu(Z) \tilde{r}_{n}^{-(1-1 / n)}\right)}{-\log \tilde{r}_{n}}=1-\frac{1}{n}-\frac{\log \mu(Z)}{\log \tilde{r}_{n}} \underset{n \rightarrow \infty}{\longrightarrow} 1 .
$$

Since $\tilde{r}_{n} \rightarrow 0$ as $n \rightarrow \infty$, this implies that that $\overline{\operatorname{dim}}_{B}(Z)=1$. Thus, $\overline{\operatorname{dim}}_{B}(Z)=1$ for any set $Z$ with $\mu(Z)>0$, and hence $\overline{\operatorname{dim}}_{B} \mu=1$ by the definition (2.1).

Now we prove that $\underline{\operatorname{dim}}_{B} \mu$, the lower box dimension of $\mu$, equals 0 . Let $G_{k}=$ $\bigcap_{n=k}^{\infty} E_{n}$. By $(\underline{3.12}), \mu\left(E_{n}\right) \geq 1-5 / 2^{n}$ for each $n$, and hence

$$
\mu\left(G_{k}\right) \geq 1-5 / 2^{k-1} \rightarrow 1 \text { as } k \rightarrow \infty .
$$

We recall that for each $n$ the set $E_{n}$ consists of $1 / s_{n}$ intervals of length at most $r_{n}$, and $\log s_{n} / \log r_{n} \rightarrow 0$ as $n \rightarrow \infty$. This implies that each $E_{n}$, and hence $G_{k}$, can be covered by at most $1 / s_{n}$ balls of diameter $r_{n}$, i.e. $N\left(G_{k}, r_{n}\right) \leq 1 / s_{n}$. Therefore,

$$
\underline{\operatorname{dim}}_{B} G_{k}=\liminf _{\varepsilon \rightarrow 0} \frac{\log N\left(G_{k}, \varepsilon\right)}{\log (1 / \varepsilon)} \leq \liminf _{n \rightarrow \infty} \frac{\log N\left(G_{k}, r_{n}\right)}{\log \left(1 / r_{n}\right)} \leq \lim _{n \rightarrow \infty} \frac{\log s_{n}}{\log r_{n}}=0
$$

for any $k>0$. Thus for any $\varepsilon>0$ there exists a set $G$ such that $\mu(G)>1-\varepsilon$ and $\underline{\operatorname{dim}}_{B} G=0$, which implies that $\underline{\operatorname{dim}}_{B} \mu=0$ by the definition (2.1). And since $0 \leq \operatorname{dim}_{H} \mu \leq \underline{\operatorname{dim}}_{B} \mu$, it follows that the Hausdorff dimension of $\mu$ is also 0 .

This completes the proof of the theorem for the case of $\beta=0$. 
The case of $0<\beta<1$.

The proof for this case uses the same approach as the proof for the case of $\beta=0$. However, some modifications are needed to ensure that the lower pointwise dimension is $\beta>0$.

Let $\gamma=(1-\beta) / 2$, then $\gamma>0$ and $\beta+\gamma / n<1$ for any $n \geq 1$. We will construct the sequences $\left\{\tau_{n}\right\}_{n=1}^{\infty}$ and $\left\{h_{n}\right\}_{n=1}^{\infty}$ inductively. Let $h_{1}$ be the identity map, $\tau_{1}$ be a rational number close to $\tau$, and $s_{1}$ be a number such that $1 / s_{1}$ is an integer and $\delta_{1}=s_{1}^{1 /(\beta+\gamma)}<s_{1} / 2$. Suppose that $\tau_{n-1}=p_{n-1} / q_{n-1}$, a function $a_{n-1}$ of period $s_{n-1}$, and hence $A_{n-1}$ and $h_{n}$ are selected. As before, we set $M_{n}=\max _{[0,1]} h_{n}^{\prime}$ and $m_{n}=\min _{[0,1]} h_{n}^{\prime}$. We choose numbers $\tau_{n}=p_{n} / q_{n}, s_{n}$ and $\delta_{n}$, and functions $a_{n}$ of a period $s_{n}$ and $A_{n}$ such that

(i) $\left|\tau-\tau_{n}\right| \leq\left|\tau-\tau_{n-1}\right|$,

(ii) $\left|\tau-\tau_{n}\right|=\left|\tau-p_{n} / q_{n}\right| \leq 1 / q_{n}^{n^{4}}$,

(iii) $q_{n} \geq \max \left\{1 / s_{n-1}, 1 / m_{n},\left(3 M_{n}\right)^{n}, c_{n}, \tilde{c}\left(h_{n}, n+1\right)\right\}$,

where $c_{n}$ is as in (3.4) and $\tilde{c}\left(h_{n}, n+1\right)$ is as in (3.7),

(iv) $s_{n}=s_{n-1} / q_{n}$, hence $s_{n} \leq s_{n-1}^{2}$ and $s_{n} \leq 1 / 2^{n}$,

(v) $s_{n} \leq 2^{-n-1} s_{n-1}$, and $s_{n}^{\gamma} \leq \min \left\{1 /\left(M_{n}+1\right)^{n},\left(m_{n} /\left(2 M_{n}\right)\right)^{n}\right\}$,

(vi) $\delta_{n}=m_{n} s_{n}^{1 /(\beta+\gamma / n)}$, hence $\delta_{n}<s_{n} / 2$,

(vii) $a_{n}$ and $A_{n}$ are as in Lemma 3.3 with $\delta=\delta_{n}$ and $s=s_{n}$.

Condition (v) will be used only in the proof of Lemma 3.5 below. Clearly, it can be satisfied by choosing a sufficiently large $q_{n}$. We note that $q_{n} \geq \tilde{c}\left(h_{n}, n+1\right) \geq$ $\|h\|_{n} \geq \max \left|h^{-1}\right|=1 / m_{n}$, and hence

$$
\frac{1}{\delta_{n}}=\frac{1}{m_{n} s_{n}^{1 /(\beta+\gamma / n)}}=\frac{q_{n}^{1 /(\beta+\gamma / n)}}{m_{n} s_{n-1}^{1 /(\beta+\gamma / n)}} \leq \frac{q_{n}^{2 /(\beta+\gamma / n)}}{m_{n}} \leq \frac{q_{n}^{2 / \beta}}{m_{n}} \leq q_{n}^{(2 / \beta)+1} \leq q_{n}^{3 / \beta} .
$$

Thus we can obtain an estimate similar to (3.9):

$$
\begin{aligned}
& d_{n}\left(f_{n+1}, f_{n}\right)=\ldots \leq 2 c_{n}\left|\tau-\tau_{n}\right| \cdot\left(\tilde{c}\left(h_{n}, n+1\right) / \delta_{n}^{(n+1)^{2}}\right)^{n+1} \leq \\
& 2 q_{n}\left(1 / q_{n}^{n^{4}}\right)\left(q_{n}\left(q_{n}^{3 / \beta}\right)^{(n+1)^{2}}\right)^{n+1} \leq 2\left(1 / q_{n}^{n^{4}}\right) q_{n}^{(3 / \beta)(n+1)^{3}+n+2} \leq 1 / 2^{n}
\end{aligned}
$$

for all sufficiently large $n$, and establish convergence of the sequence $\left\{f_{n}\right\}$ in the $C^{\infty}$ topology. The convergence of $\left\{h_{n}\right\}$ with respect to the distance $d_{0}$ can be shown as before.

We define the sets $\tilde{E}_{n}$ and $E_{n}$ be as in (3.11). The total growth of $A_{n}$ on $\tilde{E}_{n}$, and hence the total growth of $h_{n+1}$ on $E_{n}$ is

$$
\left(s_{n}-\delta_{n}\right)\left(1 / s_{n}\right)=1-\delta_{n} / s_{n} \geq 1-s_{n}^{(1 /(\beta+\gamma / n))-1} .
$$


Since $E_{n}$ consists of $1 / s_{n}$ intervals, $\max \left|h-h_{n+1}\right| \leq 2 s_{n+1}$, and $s_{n+1} \leq s_{n}^{2}$, the total growth of $h$ on $E_{n}$ is at least

$$
1-s_{n}^{(1 /(\beta+\gamma / n))-1}-4 s_{n+1} / s_{n} \geq 1-s_{n}^{(1 /(\beta+\gamma / n))-1}-4 s_{n} \geq 1-5 s_{n}^{\sigma} \geq 1-5 / 2^{n \sigma},
$$

where $\sigma=\min \{(1 /(\beta+\gamma)-1,1\}>0$. Thus, for the measure $\mu$ with distribution function $h$,

$$
\mu\left(E_{n}\right) \geq 1-5 / 2^{n \sigma}
$$

Now we will show that $\underline{d}_{\mu}(x)=\beta$ for almost all $x$. We take

$$
\begin{gathered}
r_{n}=\delta_{n} / m_{n}=s_{n}^{1 /(\beta+\gamma / n)}, \text { then } \\
r_{n}<s_{n}, \quad s_{n}=r_{n}^{\beta+\gamma / n}, \quad \text { and hence } \frac{\log s_{n}}{\log r_{n}}=\beta+\frac{\gamma}{n} .
\end{gathered}
$$

Let $x$ be a point in $E_{n}$. Then

$$
\Delta h\left(x, r_{n}\right) \geq \Delta h_{n+1}\left(x, r_{n}\right)-4 s_{n+1} \geq s_{n}-\delta_{n}-4 s_{n+1} \geq s_{n} / 2
$$

for all sufficiently large $n$. Therefore

$$
\frac{\log \Delta h\left(x, r_{n}\right)}{\log r_{n}} \leq \frac{\log \left(s_{n} / 2\right)}{\log r_{n}}=\frac{\log s_{n}}{\log r_{n}}-\frac{\log 2}{\log r_{n}}=\beta-\frac{\log 2}{\log r_{n}} \leq \beta+\frac{1}{n}
$$

since $r_{n}<s_{n} \leq 1 / 2^{n}$. It follows as before that $\underline{d}_{\mu}(x) \leq \beta$ for $\mu$-almost every $x$.

To show that $\underline{d}_{\mu}(x) \geq \beta$ we will prove that the function $h$ is Hölder continuous with exponent $\beta$. Then for any $x$ and $r>0, \Delta h(x, r) \leq C(2 r)^{\beta}$ and hence

$$
\frac{\log \mu(B(x, r))}{\log r}=\frac{\log \Delta h(x, r)}{\log r} \geq \frac{\log \left(C 2^{\beta}\right)+\beta \log r}{\log r} \underset{r \rightarrow 0}{\longrightarrow} \beta .
$$

This implies that $\underline{d}_{\mu}(x) \geq \beta$ for all $x$, and hence $\underline{d}_{\mu}(x)=\beta$ for $\mu$-almost every $x$.

Lemma 3.5. The function $h$ is Hölder continuous with exponent $\beta$.

Proof. We will show using induction that

$$
\begin{aligned}
& \left|h_{n}(x)-h_{n}(y)\right| \leq|x-y|^{\beta} \quad \text { for all } x, y \text { with }|x-y| \leq s_{n-1}, \text { and } \\
& \left|h_{n}(x)-h_{n}(y)\right| \leq\left(3-2^{-n}\right)|x-y|^{\beta} \quad \text { for all } x, y \text { with }|x-y| \geq s_{n-1} .
\end{aligned}
$$

Clearly, this is true for $h_{1}=$ Id. Suppose that it holds for $h_{n}$.

Recall that $h_{n+1}=A_{n} \circ h_{n}$, and the diffeomorphism $A_{n}$ is of the form Id $+a_{n}$, where $a_{n} \geq 0$ is a function of period $s_{n}$. It follows that $x \leq A_{n}(x) \leq x+s_{n}$ for any point $x$ in $[0,1]$. Hence, $\left|A_{n}(x)-A_{n}(y)\right| \leq|x-y|+s_{n}$ for any $x$ and $y$.

If $|x-y| \geq s_{n-1}$ we obtain

$$
\begin{aligned}
& \left|h_{n+1}(x)-h_{n+1}(y)\right|=\left|A_{n}\left(h_{n}(x)\right)-A_{n}\left(h_{n}(y)\right)\right| \leq\left|h_{n}(x)-h_{n}(y)\right|+s_{n} \\
& \leq\left(3-2^{-n}\right)|x-y|^{\beta}+2^{-n-1}|x-y|^{\beta}=\left(3-2^{-n-1}\right)|x-y|^{\beta}
\end{aligned}
$$


since by (3.14) $s_{n} \leq 2^{-n-1} s_{n-1} \leq 2^{-n-1}|x-y| \leq 2^{-n-1}|x-y|^{\beta}$.

If $s_{n} \leq|x-y| \leq s_{n-1}$ we have

$$
\left|h_{n+1}(x)-h_{n+1}(y)\right| \leq\left|h_{n}(x)-h_{n}(y)\right|+s_{n} \leq|x-y|^{\beta}+s_{n} \leq 2|x-y|^{\beta} .
$$

It follows from (3.14) and (3.17) that $r_{n}^{\gamma / n} \leq s_{n}^{\gamma / n} \leq \min \left\{1 /\left(M_{n}+1\right), m_{n} /\left(2 M_{n}\right)\right\}$. We will use this together with the fact that $\beta+\gamma / n<1$ for all $n$ in the two estimates below. Suppose that $r_{n} \leq|x-y| \leq s_{n}$. Then

$$
\begin{aligned}
& \left|h_{n+1}(x)-h_{n+1}(y)\right| \leq\left|h_{n}(x)-h_{n}(y)\right|+s_{n} \leq M_{n}|x-y|+s_{n} \\
& \quad \leq\left(M_{n}+1\right) s_{n}=\left(M_{n}+1\right) r_{n}^{\beta+\gamma / n} \leq r_{n}^{\beta} \leq|x-y|^{\beta} .
\end{aligned}
$$

Finally, for $|x-y| \leq r_{n}$ we have

$$
\begin{aligned}
& \left|h_{n+1}(x)-h_{n+1}(y)\right| \leq \max h_{n+1}^{\prime} \cdot|x-y|=\max A_{n}^{\prime} \cdot \max h_{n}^{\prime} \cdot|x-y| \\
& \leq \frac{2 s_{n}}{\delta_{n}} \cdot M_{n} \cdot|x-y|=\frac{r_{n}^{\beta+\gamma / n}}{r_{n} m_{n}} \cdot 2 M_{n} \cdot|x-y| \leq \frac{2 M_{n}}{m_{n}} \cdot|x-y|^{\beta+\gamma / n} \\
& \leq \frac{2 M_{n}}{m_{n}} \cdot r_{n}^{\gamma / n} \cdot|x-y|^{\beta} \leq|x-y|^{\beta} .
\end{aligned}
$$

Thus, each function $h_{n}$ satisfies $\left|h_{n}(x)-h_{n}(y)\right| \leq 3|x-y|^{\beta}$ for all $x$ and $y$. Since the sequence $\left\{h_{n}\right\}$ converges to $h$, it follows that

$$
|h(x)-h(y)| \leq 3|x-y|^{\beta} \quad \text { for all } x \text { and } y \text {. }
$$

The proof of the fact that $\bar{d}_{\mu}(x)=1$ for $\mu$-almost every $x$ does not require any modifications. Thus

$$
\underline{d}_{\mu}(x)=\beta \quad \text { and } \quad \bar{d}_{\mu}(x)=1 \text { for } \mu \text {-almost every } x .
$$

The same argument as for $\beta=0$ shows that $\overline{\operatorname{dim}}_{B} \mu=1$ for all $x$.

Now we show that $\operatorname{dim}_{H} \mu=\underline{\operatorname{dim}}_{B} \mu=\beta$. Since $\underline{d}_{\mu}(x)=\beta$ for almost all $x$, it follows from Theorem 2.1 that $\operatorname{dim}_{H} \mu \geq \beta$. So it remains to show that $\underline{\operatorname{dim}}_{B} \mu \leq \beta$. As before, let $G_{k}=\bigcap_{n=k}^{\infty} E_{n}$. Since $G_{k} \subset E_{n}$ and $E_{n}$ consists of $1 / s_{n}$ intervals of length at most $r_{n}$, we have $N\left(G_{k}, r_{n}\right) \leq 1 / s_{n}$. As $\log s_{n} / \log r_{n} \rightarrow \beta$ as $n \rightarrow \infty$, we obtain

$$
\underline{\operatorname{dim}}_{B} G_{k} \leq \lim _{n \rightarrow \infty} \frac{\log N\left(G_{k}, r_{n}\right)}{\log \left(1 / r_{n}\right)} \leq \lim _{n \rightarrow \infty} \frac{\log s_{n}}{\log r_{n}}=\beta
$$

for any $k>0$. It follows from (3.15) that $\mu\left(G_{k}\right) \rightarrow 1$ as $k \rightarrow \infty$, and hence $\underline{\operatorname{dim}}_{B} \mu \leq \beta$.

This completes the proof of the theorem. 
DIMENSIONAL CHARACTERISTICS OF INVARIANT MEASURES FOR CIRCLE DIFFEOS 16

\section{REFERENCES}

[1] D. Anosov, A. Katok. New Examples in Smooth Ergodic Theory. Ergodic diffeomorphisms. Transactions of the Moscow Mathematical Society, 23 (1970), 1-35.

[2] L. Barreira, Ya. Pesin, J. Schmeling. Dimension and Product Structure of Hyperbolic Measures. Annals of Math., vol. 149:3, (1999), 755-783.

[3] J. P. Eckmann, D. Ruelle. Ergodic Theory of Chaos and Strange Attractors. 3, Rev. Mod. Phys. 57 (1985), 617-656.

[4] B. Fayad, M. Saprykina. Weak mixing disc and annulus diffeomorphisms with arbitrary Liouvillean rotation number on the boundary. Ann. Sci. cole Norm. Sup. 38 (2005), no. 3, p. 339-364.

[5] B. Fayad, M. Saprykina, A. Windsor. Non-Standard Smooth Realizations of Liouville Rotations. To appear in Ergod. Theory and Dyn. Syst.

[6] M.-R. Herman. Sur la conjugaison différentiable des difféomorphismes du cercle à des rotations. Inst. Hauntes Études Sci. Publ. Math., No. 49, (1979), 5-233.

[7] A. Katok, B. Hasselblatt. Introduction to the Modern Theory of Dynamical Systems. Encyclopedia of Mathematics and its Applications, vol. 54. Cambridge University Press, London-New York, 1995.

[8] B. Kalinin, V. Sadovskaya. On pointwise dimension of nonhyperbolic measures. Ergod. Theory and Dyn. Syst. (2002) 22 no.6, 1783-1801.

[9] F. Ledrappier, M. Misiurewicz. Dimension of Invariant Measures for Maps with Exponent Zero. Ergod. Theory and Dyn. Syst. (1985) 5, 595-610.

[10] Ya. Pesin. Dimension Theory in Dynamical Systems: Contemporary Views and Applications. The University of Chicago Press, Chicago and London.

[11] L.-S. Young. Dimension, Entropy, and Lyapunov Exponents. Ergod. Theory and Dyn. Syst. 2 (1982), 109-124.

Department of Mathematics \& Statistics, University of South Alabama, Mobile, AL 36688, USA

E-mail address: sadovska@jaguar1.usouthal.edu 